\title{
Detección de déficits neuromusculares a través del análisis del patrón de salto y aterrizaje en deportistas adolescentes
}

\section{Detection of neuromuscular deficits through jumping pattern analysis in adolescent athletes}

\section{Detecção de déficits neuromusculares por meio da análise do padrão de saltos e aterrissagens em atletas adolescentes}

\author{
Benet-Vigo, A. ${ }^{1,2}$, Arboix-Alió, J. ${ }^{1}$, Montalvo, A.M. ${ }^{3}$, Myer, G.D. ${ }^{4}$ y Fort-Vanmeerhaeghe, A. ${ }^{1,2}$ \\ ${ }^{1}$ Faculty of Psychology, Education Science and Sport (FPCEE) Blanquerna, Universitat Ramon Llull, \\ (Barcelona, Spain); ${ }^{2}$ Segle XXI Female Basketball Team, Federació Catalana de Basquetbol, (Esplugues \\ de Llobregat, Spain); ${ }^{3}$ College of Health Solutions, Arizona State University, Phoenix, (AZ 85004, USA); \\ ${ }^{4}$ Division of Sports Medicine, Cincinnati Children's Hospital Medical Center, (Cincinnati, Ohio)
}

\section{RESUMEN}

El objetivo principal del presente estudio fue analizar el rendimiento de la técnica del salto y aterrizaje en deportistas adolescentes mediante la versión modificada del Tuck Jump Assessment (TJA). Como objetivo secundario se compararon los resultados obtenidos, según sexo. Se puntuó cada variable de la herramienta mediante el análisis en video de la prueba de los 75 participantes incluidos en el estudio. Las variables contempladas fueron la puntuación global del TJA y cada criterio del instrumento: 1) Valgo de la extremidad inferior en el aterrizaje; 2) Muslos no paralelos (punto máximo de salto); 3) Muslos no alineados durante la fase de vuelo; 4) Mala alineación de los pies con los hombros; 5) Posición de los pies no paralela (de la punta al talón); 6) Contacto desigual de los pies (aterrizaje asimétrico); 7) Demasiado ruido en el aterrizaje; 8) Pausa entre saltos; 9) La técnica empeora antes de los 10 segundos; 10) No aterriza en el mismo punto (salto consistente). Los valores promedios de la puntuación global de la versión modificada del TJA fueron 8.85 \pm 2.31 en el total de la muestra (8.85 \pm 2.63 hombres vs. $8.86 \pm 2.14$ mujeres). Los resultados de la comparación según sexo indicaron diferencias significativas en el criterio $1(\chi 2=10.288 ; p<0.01)$, en el criterio $2(\chi 2=20.788 ; p<0.01)$, en el criterio 4 $(\chi 2=22.587 ; p<0.01)$ y en el criterio $10(\chi 2=8.883 ; p<0.01)$. En consecuencia, el análisis del patrón de salto y aterrizaje permite identificar alteraciones neuromusculares y biomecánicas del patrón de salto-aterrizaje.

Palabras clave: Herramienta de valoración; LCA; Tuck Jump Assessment; prevención

\section{ABSTRACT}

The main objective of the present study was to analyze the performance of the jump and landing technique in adolescent team sports athletes through the modified version of the Tuck Jump Assessment, (TJA). As a secondary objective, the results obtained were compared, according to sex. Each outcome measure of the tool was scored 


\section{Detección de déficits neuromusculares en deportistas adolescentes}

through video analysis of the test of the 75 participants included in the study. The main outcome measures were the total score of TJA and each of the ten items: Knee valgus at landing (1), thighs do not reach parallel (2), thighs not equal side-to-side (3), foot placement not shoulder width apart (4), foot placement not parallel (5), foot contact timing not equal (6), excessive landing contact noise (7), pause between jumps (8), technique declines prior to ten seconds (9), and does not land in same footprint (10). The variables contemplated were the global score of the TJA and the ten criteria of the instrument. The average values of the global score of the modified version of the TJA were $8.85 \pm 2.31$ in the total sample $(8.85 \pm 2.63$ men vs. $8.86 \pm 2.14$ women). The results of the gender comparison indicated significant differences in item $1(\chi 2=10.288 ; p<0.01)$, item $2(\chi 2=20.788 ; p<0.01)$, item $4(\chi 2=22.587$; $p<0.01)$ and item $10(\chi 2=8.883 ; p<0.01)$. Consequently, the analysis of the jump and landing pattern makes it possible to identify neuromuscular and biomechanical alterations of the jump-landing pattern.

Keywords: Screening tool; ACL; Tuck Jump Assessment; prevention

\section{RESUMO}

O objetivo principal do presente estúdio fue analisar o rendimiento da técnica do salto e aterrissagem em deportistas adolescentes mediante a versão modificada do Tuck Jump Assessment (TJA). Como objetivo secundário se comparar os resultados obtidos, según sexo. Se puntuó cada variável do herramienta mediante a análise no vídeo da prueba dos 75 participantes incluidos no estudio. As variáveis contempladas na versão global do TJA e cada critério do instrumento: 1) Valgo de la extremidad inferior en el aterrizaje; 2) Muslos no paralelos (ponto máximo de salto); 3) Muslos no alineados durante la fase de vuelo; 4) Mala alineación de los pies con los hombros; 5) Posición de los pies no paralela (de la punta al talón); 6) Contacto desigual de los pies (aterrizaje asimétrico); 7) Demasiado ruido en el aterrizaje; 8) Pausa entre saltos; 9) La técnica empeora antes de los 10 segundos; 10) No aterriza en el mismo punto (salto consistente). Os valores prometidos da versão global modificada do TJA fueron $8.85 \pm 2.31$ no total da muestra $(8.85 \pm 2.63$ hombres vs. $8.86 \pm 2.14$ mujeres $)$. Os resultados da comparação segundo sexo indicaron diferencias relevantes no critério $1(\chi 2=10.288 ; p<0.01)$, no critério $2\left(\chi^{2}=20.788 ; p<0.01\right)$, no critério $4\left(\chi^{2}=22.587 ; p<0.01\right)$ e o critério $10\left(\chi^{2}=8.883 ; p<0.01\right)$. Na sequência, a análise do patrón de salto y aterrizaje permite identificar alterações neuromusculares y biomecánicas del patrón de salto-aterrizaje.

Palavras chave: Ferramenta de avaliação; LCA; Tuck Jump Assessment; prevenção

\section{INTRODUCCION}

En los últimos años, el aumento de la práctica y la especialización precoz en los deportes de equipo se ha asociado al incremento de la incidencia lesiva (Myer et al., 2015), destacando por su severidad la lesión del ligamento cruzado anterior (LCA) (Myer et al., 2011b). Esta incidencia lesiva se acentúa cuando se analizan datos epidemiológicos en mujeres deportistas y aún más, en la etapa de la adolescencia, alcanzando una prevalencia superior a 1.5 respecto a deportistas masculinos (Agel et al., 2016; Gornitzky et al., 2016). Las lesiones del LCA implican una baja deportiva mínima de entre 6 y 9 meses y, en la mayoría de los casos, requieren de una intervención quirúrgica (Yanguas-Leyes et al., 2011). Además, pueden conllevar graves consecuencias, como un elevado riesgo de recaída durante los primeros meses de reaparición deportiva o bien, a largo plazo, la aparición de procesos degenerativos de tipo artrósico de la rodilla lesionada (Lohmander et al., 2007; Shimokochi y Shultz, 2008). Por estos motivos, resulta fundamental promover estrategias para prevenir lesiones deportivas en esta población de riesgo.

Existe un gran acuerdo entre la comunidad científica en que el riesgo de sufrir una lesión de LCA es multifactorial y por ello, es necesario profundizar en el conocimiento de su etiología (Hewett et al., 2010). La mayor comprensión del mecanismo lesivo y de los factores de riesgo permite optimizar las estrategias de prevención de dicha lesión (Bahr y Krosshaug, 2005; Sugimoto et al., 2015). En este sentido, la mayoría de las lesiones de LCA sin contacto en mujeres que participan en deportes de equipo se asocia a alteraciones en los patrones de movimiento durante 


\section{Benet-Vigo, A. Arboix-Alió, J., Montalvo, A.M., Myer, G.D. y Fort-Vanmeerhaeghe, A.}

acciones de desaceleración, recepciones de salto y/o cambios de dirección (Fort-Vanmeerhaeghe et al., 2016). Estas alteraciones del movimiento desencadenan en una traslación anterior de la tibia y la consecuente rotura del LCA (Hewett et al., 2016). En el caso de la habilidad de salto, Hewett et al., (2009) observaron mediante el análisis de vídeo cuatro mecanismos lesivos comunes durante la fase de aterrizaje clasificados en: a) valgo de rodilla durante el aterrizaje; b) rodilla lesionada cerca de la extensión completa al contactar con el suelo; c) una gran parte del peso recae sobre la extremidad lesionada; y d) el tronco tiende a inclinarse lateralmente.

Asimismo, se han hecho avances significativos en una mayor comprensión de los factores de riesgo de lesión del LCA, clasificados en las siguientes categorías: ambientales, anatómicos, hormonales y neuromusculares (Griffin et al., 2006); siendo estos últimos, modificables mediante el entrenamiento (Hewett et al., 2006). Cabe destacar que los patrones de movimiento lesivos descritos anteriormente se asocian a múltiples desequilibrios neuromusculares y biomecánicos: a) una mayor dependencia del control neuromuscular en el plano frontal en comparación con el plano sagital (valgo dinámico o dominio ligamentoso); b) una estrategia dominante de la musculatura del cuádriceps versus la isquiosural para estabilizar la articulación de la rodilla (dominancia del cuádriceps); c) mayor fuerza, coordinación y equilibrio en la extremidad dominante (dominio de una pierna); d) disminución de la propiocepción y la estabilidad del tronco (dominio del tronco); e) fatiga neuromuscular que provoca la disminución de la estabilidad articular y, f) déficits en los mecanismos de pre activación o vigilancia muscular (Myer et al., 2011a; Fort-Vanmeerhaeghe et al., 2017).

Tradicionalmente, para evaluar el riesgo de lesión del LCA se ha utilizado la captura de movimiento tridimensional (variables cinéticas y cinemáticas) tanto en tareas de aterrizaje del salto (Hewett et al., 2006) como en cambios de dirección (Fox, 2018). Este tipo de análisis, sin embargo, requiere una amplia formación de los evaluadores y, elevados recursos económicos, motivo por el que habitualmente se ha desarrollado en entornos clínicos, a pesar de su creciente aplicación en la práctica físico-deportiva. No obstante, investigaciones recientes han conseguido desarrollar herramientas más rentables y prácticas que permiten detectar los factores de riesgo de lesión neuromusculares y biomecánicos de la lesión del LCA (Crossley et al., 2011; Padua et al., 2011). Una alternativa a este tipo de análisis, que no requiere de alta tecnología y ha alcanzado gran popularidad en los últimos tiempos, es el Tuck Jump Assessment (TJA) (Myer et al., 2008a). El TJA consiste en la ejecución de saltos continuados alcanzando la máxima altura posible durante diez segundos, e implica el análisis de diez criterios de valoración cualitativos y dicotómicos. Estos diez criterios permiten relacionar los desequilibrios biomecánicos y neuromusculares detectados durante el salto vertical asociados a los factores de riesgo de lesión del LCA (Fort-Vanmeerhaeghe et al., 2019) citados anteriormente. Pese a que se trata de un instrumento de valoración de carácter subjetivo, se acerca a la realidad de los deportes de equipo, donde se combinan elementos como los ciclos de estiramiento acortamiento de tipo reflejo juntamente con la presencia de fatiga. No se trata de una herramienta de predicción de las lesiones como tal, pero sí es un instrumento que permite identificar alteraciones del patrón técnico de salto y aterrizaje, relacionados con el riesgo de lesión del LCA (Myer et al., 2008b). Aunque su validez no ha sido aún demostrada, ha reportado una excelente fiabilidad inter e intra observador (Fort-Vanmeerhaeghe et al., 2017; Herrington et al., 2013; Read et al., 2016) siendo actualmente una herramienta muy utilizada tanto en el campo de la rehabilitación deportiva como para la prevención de lesiones deportivas.

Recientemente, se ha publicado una versión modificada del TJA (Fort-Vanmeerhaeghe et al., 2017) que amplía la escala de puntuación de los diez criterios de la versión original. Mientras la versión original centra la evaluación de los criterios en una puntuación dicotómica, la propuesta modificada propone ampliar la puntuación a tres criterios $(0$ : alteración inexistente del patrón técnico del salto y aterrizaje; 1: alteración leve del patrón técnico del salto; 2: alteración severa y/o persistente del patrón técnico del salto). Esta modificación en la escala de valoración está mostrando una buena aceptación entre la comunidad científica, aplicando esta herramienta en estudios de asociación de los factores de riesgo de lesión de las extremidades inferiores (Arundale et al., 2020a; Lindblom et al., 2020; Smith et al., 2017; Standing y Maulder, 2019). Si bien 


\section{Detección de déficits neuromusculares en deportistas adolescentes}

existen algunos estudios que aportan valores normales de los criterios de puntuación del TJA (Arundale et al., 2020a; Arundale et al., 2020b; Lindblom et al., 2020), contemplando distintas poblaciones en cuanto a sexo o modalidades deportivas, existen escasas publicaciones que aporten los valores normales de la versión modificada del TJA (Fort-Vanmeerhaeghe, et al., 2019; Standing y Maulder, 2019). Por este motivo, se plantean los siguientes objetivos: 1) cuantificar y describir los valores normales de la versión modificada del TJA en deportistas adolescentes de tecnificación; 2) comparar los valores de la versión modificada del TJA según sexo.

\section{MÉTODO}

\section{Participantes}

La muestra estuvo configurada por un total de 75 jugadores de deportes de equipo, 26 hombres (11 de balonmano y 15 de voleibol) y 49 mujeres (22 de baloncesto, 14 de balonmano y 13 de voleibol), de edades compuestas entre los 14 y 18 años, todos ellos procedentes de un centro de tecnificación deportiva (Residencia Joaquim Blume, Esplugues de Llobregat). Los criterios de exclusión fueron padecer o haber padecido una lesión previa en los últimos doce meses o padecer una enfermedad durante la prueba que impidiesen su realización al máximo rendimiento. La tabla 1 proporciona las características de los sujetos. La carga de entrenamientos semanal consistió en 8-10 sesiones por semana con 90-120 minutos por sesión, más un partido de fin de semana, completando aproximadamente 12-18 horas de entrenamiento y competición por semana. Antes del comienzo del estudio, los sujetos y sus padres recibieron información detallada escrita y verbal sobre los posibles riesgos y beneficios asociados con las pruebas. Se obtuvo el consentimiento informado por escrito y el asentimiento tanto de los padres/tutores como de los participantes, respectivamente. El Comité de Ética del Consejo Catalán del Deporte aprobó el estudio (07/2017 / CEICEGC) y se ajustó a las recomendaciones de la Declaración de Helsinki.

\begin{tabular}{lccccc} 
Tabla 1. Características de los sujetos. Datos expresados en media \pm SD. \\
\hline & $\begin{array}{c}\text { Edad } \\
\text { cronológica } \\
\text { (años) }\end{array}$ & $\begin{array}{c}\text { Velocidad } \\
\text { máxima de } \\
\text { crecimiento* } \\
\text { (años) }\end{array}$ & Altura (m) & $\begin{array}{c}\text { Peso corporal } \\
\text { (kg) }\end{array}$ & $\begin{array}{c}\text { Masa } \\
\text { corporal (\%) }\end{array}$ \\
\hline Hombres (n=26) & $16.08 \pm 1.08$ & $1.58 \pm 2.26$ & $1.85 \pm 0.08$ & $73.93 \pm 13.18$ & $21.45 \pm 2.94$ \\
\hline Balonmano (n=11) & $16.88 \pm 0.56$ & $0.57 \pm 2.55$ & $1.83 \pm 0.08$ & $78.59 \pm 15.30$ & $23.27 \pm 2.72$ \\
Voleibol (n=15) & $15.50 \pm 1.01$ & $2.32 \pm 1.76$ & $1.87 \pm 0.08$ & $70.51 \pm 10.64$ & $20.12 \pm 2.37$ \\
\hline Mujeres (n=49) & $15.74 \pm 1.15$ & $3.51 \pm 0.88$ & $1.78 \pm 0.08$ & $66.49 \pm 9.08$ & $20.87 \pm 2.01$ \\
\hline Baloncesto (n=22) & $16.15 \pm 1.21$ & $4.01 \pm 0.9$ & $1.82 \pm 0.07$ & $71.15 \pm 9.71$ & $21.33 \pm 2.17$ \\
Balonmano (n=14) & $15.25 \pm 0.96$ & $2.9 \pm 0.62$ & $1.73 \pm 0.06$ & $60.82 \pm 6.09$ & $20.32 \pm 1.76$ \\
Voleibol (n=13) & $15.57 \pm 1.07$ & $3.32 \pm 0.57$ & $1.77 \pm 0.05$ & $64.72 \pm 6.61$ & $20.67 \pm 1.94$ \\
\hline Total (n=75) & $15.86 \pm 1.13$ & $2.84 \pm 1.75$ & $1.81 \pm 0.08$ & $69.07 \pm 11.17$ & $21.07 \pm 2.34$ \\
\hline
\end{tabular}

IMC: Índice de Masa Corporal; * Estimación de la edad biológica (Mirwald et al., 2002)

\section{Procedimiento}

Todos participantes del estudio realizaron una sesión de familiarización sobre el TJA, una semana antes de la fecha de registro de la prueba. Para una mayor comprensión del TJA, se mostró un video con un ejemplo de un buen patrón de salto y aterrizaje. En la misma sesión, se tomaron las medidas antropométricas y se recopilaron los datos acerca del historial medico lesivo de los participantes.

Para realizar el TJA fue necesario preparar la marca dónde se ejecutaría el salto y situar los dispositivos móviles que grabaron la prueba (Figura 1). El día de ejecución de la prueba, los sujetos realizaron un calentamiento neuromuscular, que consistió en: movimientos multidireccionales, ejercicios de fuerza y de movilidad articular y desplazamientos progresivos con cambios de dirección. Después del calentamiento general, se permitió a los participantes, realizar de 3 a 5 ensayos de práctica para garantizar una interpretación precisa del salto (FortVanmeerhaeghe et al., 2017). Antes de la ejecución de la prueba, mientras los participantes lograban la recuperación completa de los ensayos, se repasaron las instrucciones para la realización de la prueba TJA fueron: 1) saltos máximos y continuos, 2) levantando las rodillas a la altura de la cadera y 3 ) aterrizando en el mismo punto con los pies separados a la altura de los hombros.

Para valorar con precisión los ítems de la prueba, se pidió a los sujetos que vistiesen con pantalones cortos de tal forma que las rodillas fuesen visibles y que calzaran zapatillas deportivas. 


\section{Benet-Vigo, A. Arboix-Alió, J., Montalvo, A.M., Myer, G.D. y Fort-Vanmeerhaeghe, A.}

Figura 1. Disposición de los dispositivos móviles para ejecutar la grabación (a) y rectángulo de la prueba Tuck Jump (b) (adaptado de FortVanmeerhaeghe et al., 2017). A) Los individuos que registraron la prueba se situaron sobre la marca de 3 metros en posición de caballero (el dispositivo móvil debe quedar aproximadamente a la altura de la cadera del sujeto) manteniendo el dispositivo móvil en formato vertical durante la grabación.

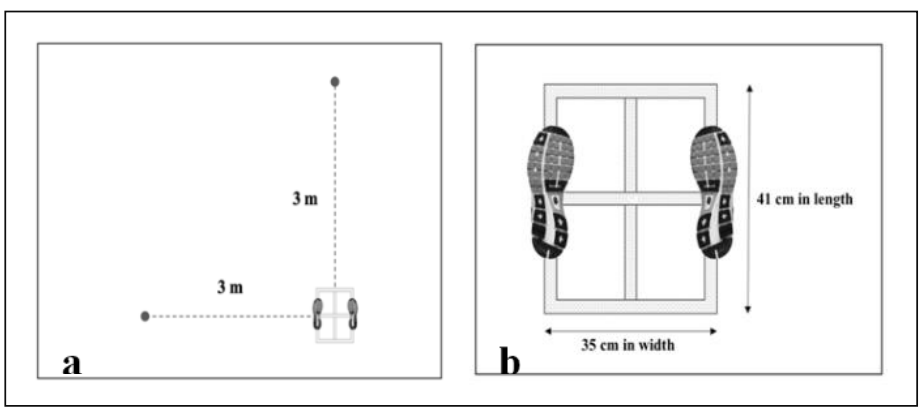

Figura 1. Disposición de los dispositivos móviles para ejecutar la grabación (a) y rectángulo de la prueba Tuck Jump (b) (adaptado de Fort-Vanmeerhaeghe y col., 2017). A) Los individuos que registraron la prueba se situaron sobre la marca de 3 metros en posición de caballero (el dispositivo móvil debe quedar aproximadamente a la altura de la cadera del sujeto) manteniendo el dispositivo móvil en formato vertical durante la grabación

Una vez registrados los videos de los sujetos, dos evaluadores, especialistas en entrenamiento de fuerza con más de cinco años de experiencia y con experiencia previa en las valoraciones del TJA modificado, analizaron y puntuaron de forma independiente cada uno de los criterios de la versión modificada del TJA. En caso de desacuerdo en la puntuación asignada entre los evaluadores, un tercer evaluador con mayor experiencia decidía la puntuación final. Se visualizaron los videos en ambos planos (vistas sagital y frontal), usando un software de video gratuito (QuickTime Player 7.6.6.) y también se permitió reproducir los videos tantas veces como fuese necesario y a diferentes velocidades.

\section{Puntuación}

Los ítems de puntuación descritos en la Figura 2 y en la Tabla 2, se clasifican en las tres fases del patrón de salto y aterrizaje: a) la acción de las rodillas y los muslos, b) la acción de los pies durante la fase de contacto y, c) el análisis de la técnica pliométrica del salto.

La primera, centrada en la acción de las rodillas y los muslos, agrupa los criterios de puntuación: 1) Valgo de la extremidad inferior en el aterrizaje, 2) Muslos no paralelos (punto máximo de salto) y 3) Muslos no alineados durante la fase de vuelo. A continuación, se analiza la acción de los pies durante la fase de contacto y, agrupa los criterios: 4) Mala alineación de los pies con los hombros, 5) Posición de los pies no paralela (de la punta al talón), 6) Contacto desigual de los pies (aterrizaje asimétrico) y 7) Demasiado ruido en el aterrizaje. La última fase se centra en el análisis de la técnica pliométrica del salto, agrupando los tres últimos criterios: 8) Pausa entre saltos, 9) La técnica empeora antes de los 10 segundos, 10) No aterriza en el mismo punto (salto consistente) y finalmente la puntuación global de la prueba, que se obtiene del sumatorio de la puntuación de los diez criterios. El anexo 1 incluye una ampliación de la descripción de cada criterio de puntuación y un enlace a un contenido videográfico que permite clarificar las alteraciones del patrón de salto y aterrizaje.

Para puntuar un ítem, la alteración del patrón debe producirse 3 o más ocasiones durante toda la prueba, invalidando el primer y el último salto. Cabe destacar que hay ítems que se valoran analizando la técnica de salto desde el plano frontal (Ítems 1, 3, 4, 6, y 9), otros que se valoran desde el plano sagital (Ítems $2 \mathrm{y}$ $5)$ y otros que pueden analizarse desde ambos planos (7, 8 y 10).

\section{Análisis estadístico}

Se utilizaron métodos estadísticos descriptivos para describir la muestra y para calcular los promedios y desviaciones estándar de las diferentes variables analizadas. Se comprobó la normalidad de la muestra mediante la prueba Kolmogorov-Smirnov.

Asimismo, se calcularon los coeficientes de concordancia intraclase $(\mathrm{CCI})$ del análisis de varianza de medidas repetidas para determinar la fiabilidad inter evaluador para la puntuación total del TJA modificado. En este caso, la significación clínica se definió como mala para un CCI por debajo de 0.4 , de regular a bueno de 0.40 a 0.75 y excelente de 0.75 o superior (Fleiss, 1986). 


\section{Detección de déficits neuromusculares en deportistas adolescentes}

Tabla 2. Criterios de puntuación de la propuesta modificada del Tuck Jump Assessment (adaptado de Fort-Vanmeerhaeghe y col., 2017)

\begin{tabular}{|c|c|c|c|c|}
\hline CRITERIO & $\begin{array}{l}\text { PLANO DE } \\
\text { OBSERVACIÓN }\end{array}$ & INEXISTENTE (0) & LEVE (1) & SEVERA (2) \\
\hline $\begin{array}{l}\text { 1. VALGO DE LA EXTREMIDAD } \\
\text { INFERIOR EN EL ATERRIZAJE }\end{array}$ & Frontal & Valgo inexistente & Valgo moderado & Valgo acentuado, las dos rodillas se tocan \\
\hline $\begin{array}{l}\text { 2. MUSLOS NO PARALELOS } \\
\text { (PUNTO MÁXIMO DE SALTO) }\end{array}$ & Sagital & $\begin{array}{l}\text { La rodilla se muestra al mismo nivel } \\
\text { o por encima del muslo }\end{array}$ & $\begin{array}{l}\text { El punto medio de la rodilla se muestra } \\
\text { sensiblemente por debajo del punto } \\
\text { medio del muslo. }\end{array}$ & $\begin{array}{l}\text { Las rodillas están claramente por debajo } \\
\text { de los glúteos }\end{array}$ \\
\hline $\begin{array}{l}\text { 3. MUSLOS NO ALINEADOS } \\
\text { DURANTE LA FASE DE VUELO }\end{array}$ & Frontal & $\begin{array}{l}\text { Los muslos están correctamente } \\
\text { alineados }\end{array}$ & $\begin{array}{l}\text { Los muslos se muestran sensiblemente } \\
\text { desalineados }\end{array}$ & $\begin{array}{l}\text { Los muslos se muestran completamente } \\
\text { desalineados (una rodilla por encima de } \\
\text { la otra) }\end{array}$ \\
\hline $\begin{array}{l}\text { 4. MALA ALINEACIÓN DE LOS } \\
\text { PIES CON LOS HOMBROS }\end{array}$ & Frontal & $\begin{array}{l}\text { Los hombros se muestran } \\
\text { correctamente alineados con los } \\
\text { pies. }\end{array}$ & $\begin{array}{l}\text { Los pies se muestran sensiblemente } \\
\text { más cerrados que los pies }\end{array}$ & $\begin{array}{l}\text { Hay contacto entre los pies en la } \\
\text { recepción del salto }\end{array}$ \\
\hline $\begin{array}{l}\text { 5. POSICIÓN DE LOS PIES NO } \\
\text { PARALELA (DE LA PUNTA AL } \\
\text { TALÓN) }\end{array}$ & Sagital & $\begin{array}{l}\text { La punta y el talón están } \\
\text { completamente en paralelo. }\end{array}$ & $\begin{array}{l}\text { La punta de un pie se muestra } \\
\text { sensiblemente por delante o por detrás } \\
\text { respecto al otro pie. }\end{array}$ & $\begin{array}{l}\text { La punta de un pie se muestra claramente } \\
\text { por delante o por detrás respecto al otro } \\
\text { pie (permite ver maléolo en el plano } \\
\text { sagital) }\end{array}$ \\
\hline $\begin{array}{l}\text { 6. CONTACTO DESIGUAL DE LOS } \\
\text { PIES (ATERRIZAJE ASIMÉTRICO) }\end{array}$ & Frontal & $\begin{array}{l}\text { La recepción del salto se efectúa al } \\
\text { mismo tiempo con ambos pies }\end{array}$ & $\begin{array}{l}\text { La recepción del salto es } \\
\text { sensiblemente asimétrica, un pie } \\
\text { aterriza poco antes que el otro }\end{array}$ & $\begin{array}{l}\text { La recepción del salto es claramente } \\
\text { asimétrica, un pie aterriza antes que el } \\
\text { otro (se aprecia con el sonido) }\end{array}$ \\
\hline $\begin{array}{l}\text { 7. DEMASIADO RUIDO EN EL } \\
\text { ATERRIZAJE }\end{array}$ & Frontal y Sagital & $\begin{array}{l}\text { Recepción del salto sin ruido. Sólo } \\
\text { efectúa el contacto con la punta de } \\
\text { los pies (hasta metatarso) }\end{array}$ & $\begin{array}{l}\text { Recepción del salto con leve ruido. Se } \\
\text { efectúa el contacto con prácticamente } \\
\text { todo el pie, sin apoyar el talón. }\end{array}$ & $\begin{array}{l}\text { Ruido pronunciado en la recepción del } \\
\text { salto. Ambos talones contactan con el } \\
\text { suelo en el aterrizaje. }\end{array}$ \\
\hline 8. PAUSA ENTRE SALTOS & Frontal y Sagital & Saltos reactivos y reflejos & Se aprecia una breve pausa entre saltos & $\begin{array}{l}\text { Doble contacto en la recepción del salto. } \\
\text { Existe una pausa claramente identificable. }\end{array}$ \\
\hline $\begin{array}{l}\text { 9. LA TÉCNICA EMPEORA ANTES } \\
\text { DE LOS } 10 \text { SEGUNDOS }\end{array}$ & Frontal & $\begin{array}{l}\text { No existe alteración en la técnica de } \\
\text { salto durante toda la prueba. }\end{array}$ & $\begin{array}{l}\text { La técnica empeora a partir del } \\
\text { segundo cinco }\end{array}$ & $\begin{array}{l}\text { La técnica empeora antes del Segundo } \\
\text { cinco }\end{array}$ \\
\hline $\begin{array}{l}\text { 10. NO ATERRIZA EN EL MISMO } \\
\text { PUNTO (SALTO CONSISTENTE) }\end{array}$ & Frontal y Sagital & $\begin{array}{l}\text { Se aterriza en la marca inicial, } \\
\text { siempre en el mismo punto }\end{array}$ & $\begin{array}{l}\text { No se aterriza en el mismo punto, pero } \\
\text { sí dentro de la marca rectangular }\end{array}$ & $\begin{array}{l}\text { Se aterriza con los pies completamente } \\
\text { fuera de la marca rectangular }\end{array}$ \\
\hline
\end{tabular}

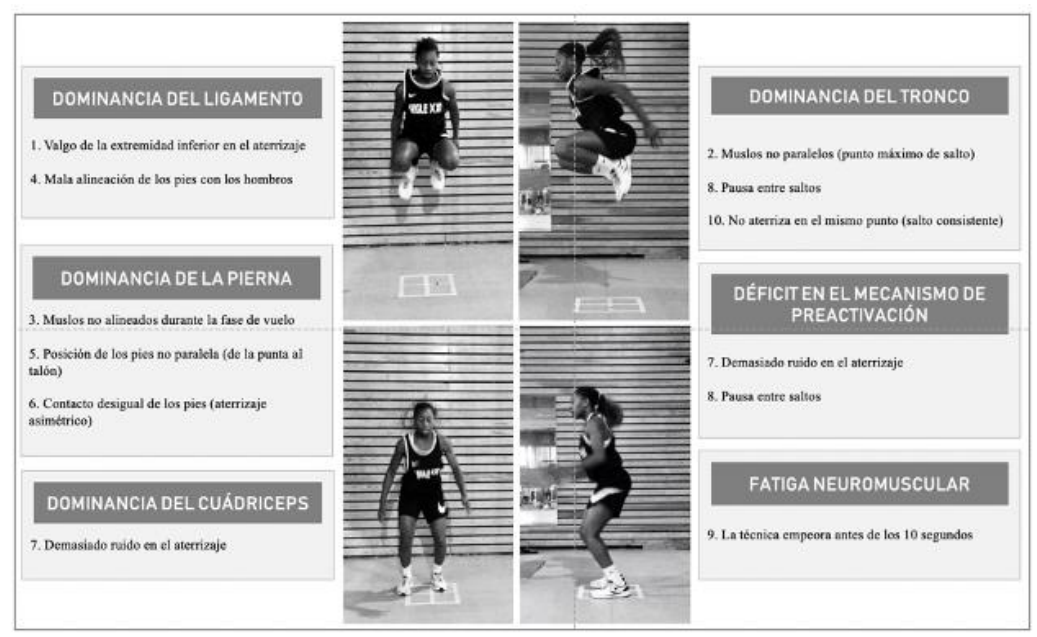

Figura 2. Relación existente entre los criterios de valoración del Tuck Jump Assessment y los factores neuromusculares asociados (adaptado de Fort-Vanmeerhaeghe y col., 2017).

Con el objetivo de identificar qué participantes obtuvieron puntuaciones globales más altas que el promedio del grupo se calculó el Z-Score (Dos'Santos et al., 2020). En este sentido, se clasificaron los participantes según las puntuaciones globales de la prueba: "pequeñas a moderadas" (promedio de puntuaciones globales de los participantes $+(0.2 \times$ desviación estándar entre sujetos)) y "altas a extremas" (promedio de puntuaciones globales de los participantes $+(1.0 \times$ desviación estándar entre sujetos). 


\section{Benet-Vigo, A. Arboix-Alió, J., Montalvo, A.M., Myer, G.D. y Fort-Vanmeerhaeghe, A.}

Para detectar diferencias según sexos, se empleó la prueba de Chi-cuadrado $(\chi 2)$ y tablas de contingencia. Adicionalmente, se utilizó la prueba tstudent para muestras relacionadas para establecer si existían diferencias significativas según sexo en la puntuación global obtenida. El análisis estadístico se realizó con el programa estadístico SPSS (Versión 20.0 para Windows; SPSS Inc., Chicago, IL, USA). El nivel de significación se fijó en 0.05 .

\section{Resultados}

Los resultados obtenidos de la prueba de concordancia intraclase (CCI) fueron excelentes en cada uno de los criterios, consiguiendo un acuerdo absoluto en la valoración de los criterios 9) $L a$ técnica empeora antes de los 10 segundos y 10) No aterriza en el mismo punto (salto consistente) (Tabla $3)$.

\begin{tabular}{lll}
\multicolumn{2}{l}{ Tabla 3. Valores de concordancia intraclase entre evaluadores } \\
\hline & Criterio & ICC $(\mathbf{9 5} \% \mathbf{C I})$ \\
\hline Acción de las & 1. Valgo de la extremidad inferior en el aterrizaje & $0.98(0.97-0.99)$ \\
rodillas y los & 2. Muslos no paralelos (punto máximo de salto) & $0.98(0.96-0.99)$ \\
muslos & 3. Muslos no alineados durante la fase de vuelo & $0.95(0.92-0,97)$ \\
\hline Acción de los & 4. Mala alineación de los pies con los hombros & $0.98(0.97-0.98)$ \\
pies durante & 5. Posición de los pies no paralela (de la punta al talón) & $0.88(0.81-0.93)$ \\
la fase de & 6. Contacto desigual de los pies (aterrizaje asimétrico) & $0.99(0.98-0.99)$ \\
contacto & 7. Demasiado ruido en el aterrizaje & $0.96(0.94-0.98)$ \\
\hline Técnica & 8. Pausa entre saltos & $0.92(0.87-0.95)$ \\
pliométrica del & 9. La técnica empeora antes de los 10 segundos & 1 \\
salto & 10. No aterriza en el mismo punto (salto consistente) & 1 \\
\hline
\end{tabular}

Los valores promedios obtenidos de la puntuación global de la versión modificada de prueba TJA fueron de $8.85 \pm 2.31$ para el total de la muestra. Se obtuvieron valores globales similares sin observar diferencias significativas entre sexos $(8.85 \pm 2.63$ en hombres vs. $8.86 \pm 2.14$ en mujeres; $p=0.42$; ES $=$ 0.004). Asimismo, en la comparativa según el sexo y las modalidades deportivas, tampoco se apreciaron diferencias significativas para hombres entre balonmano y voleibol ( $p=0.15$; ES $=0.39)$ ni para mujeres entre baloncesto, balonmano y voleibol $\left(\mathrm{F}_{(2,46)}=1.24 ; p=0.54 ; \eta_{\mathrm{p}}{ }^{2}=0.27\right)$, pero se observó que, los deportistas de voleibol, tanto hombres (9.27 \pm 3.03 ) como mujeres $(9.38 \pm 1.39)$, obtuvieron valores promedios más altos respecto a los deportistas de baloncesto $(8.55 \pm 2.61$ mujeres $)$ y balonmano $(8.27 \pm 1.95$ hombres vs $8.86 \pm 1.92$ mujeres).
La figura 3 muestra la puntuación global de cada participante y a la vez permite clasificarlos según el promedio del grupo en "pequeñas a moderadas" (zscore $=9.31)$ y en "altas a extremas" (z-score = 11.16). En la muestra del presente estudio podemos observar que 7 participantes obtuvieron puntuaciones clasificadas según "altas a extremas" y 19 participantes se situaron en el umbral de "pequeñas a moderadas".

En la comparativa de los resultados según sexo, se apreciaron diferencias significativas en los criterios valgo de la extremidad inferior en el aterrizaje (criterio 1) $(\chi 2=10.29 ; p<0.01)$, muslos no paralelos (criterio 2) $(\chi 2=20.79 ; p<0.01)$, mala alineación de los pies con los hombros (criterio 4) $(\chi 2$ $=22.59 ; p<0.01) \mathrm{y}$, no aterriza en el mismo punto (salto consistente) (criterio 10) $(\chi 2=8.88 ; p<0.01)$ (Tabla 4).

\begin{tabular}{|c|c|c|c|c|c|c|c|c|c|c|c|c|c|}
\hline \multirow{3}{*}{ Criterio de puntuación } & \multicolumn{6}{|c|}{ Hombres } & \multicolumn{6}{|c|}{ Mujeres } & \multirow{3}{*}{$p$} \\
\hline & \multicolumn{2}{|r|}{0} & \multicolumn{2}{|r|}{1} & \multicolumn{2}{|r|}{2} & \multicolumn{2}{|r|}{$\mathbf{0}$} & \multicolumn{2}{|r|}{1} & \multicolumn{2}{|r|}{2} & \\
\hline & $\mathbf{n}$ & $\%$ & $n$ & $\%$ & $\mathrm{n}$ & $\%$ & $n$ & $\%$ & $n$ & $\%$ & $\mathbf{n}$ & $\%$ & \\
\hline $\begin{array}{l}\text { 1. Valgo de la } \\
\text { extremidad* }\end{array}$ & 14 & $53.85 \%$ & 5 & $19.23 \%$ & 7 & $26.92 \%$ & 11 & $22.45 \%$ & 27 & $55.10 \%$ & 11 & $22.45 \%$ & 0.01 \\
\hline $\begin{array}{l}\text { 2. Muslos no } \\
\text { paralelos* }\end{array}$ & 20 & $76.92 \%$ & 4 & $15.38 \%$ & 2 & $7.69 \%$ & 11 & $22.45 \%$ & 25 & $51.02 \%$ & 13 & $26.53 \%$ & 0.00 \\
\hline $\begin{array}{l}\text { 3. Muslos no alineados } \\
\text { durante la fase de vuelo }\end{array}$ & 0 & $0.00 \%$ & 23 & $88.46 \%$ & 3 & $11.54 \%$ & 0 & $0.00 \%$ & 47 & $95.92 \%$ & 2 & $4.08 \%$ & 0.22 \\
\hline $\begin{array}{l}\text { 4. Mala alineación de } \\
\text { los pies con los } \\
\text { hombros" }\end{array}$ & 2 & $7.69 \%$ & 20 & $76.92 \%$ & 4 & $15.38 \%$ & 29 & $59.18 \%$ & 20 & $40.82 \%$ & 0 & $0.00 \%$ & 0.00 \\
\hline $\begin{array}{l}\text { 5. Posición de los pies } \\
\text { no paralela }\end{array}$ & 0 & $0.00 \%$ & 24 & $92.31 \%$ & 2 & $7.69 \%$ & 0 & $0.00 \%$ & 42 & $85.71 \%$ & 2 & $4.08 \%$ & 0.51 \\
\hline $\begin{array}{l}\text { 6. Contacto desigual de } \\
\text { los pies }\end{array}$ & 15 & $57.69 \%$ & 10 & $38.46 \%$ & 1 & $3.85 \%$ & 29 & $59.18 \%$ & 19 & $38.78 \%$ & 1 & $2.04 \%$ & 0.90 \\
\hline $\begin{array}{l}\text { 7. Demasiado ruido en } \\
\text { el aterrizaje }\end{array}$ & 3 & $11.54 \%$ & 19 & $73.08 \%$ & 4 & $15.38 \%$ & 1 & $2.04 \%$ & 35 & $71.43 \%$ & 13 & $26.53 \%$ & 0.15 \\
\hline 8. Pausa entre saltos & 3 & $11.54 \%$ & 23 & $88.46 \%$ & 0 & $0.00 \%$ & 9 & $18.37 \%$ & 39 & $79.59 \%$ & 1 & $2.04 \%$ & 0.55 \\
\hline $\begin{array}{l}\text { 9. La técnica empeora } \\
\text { antes de los } 10 \\
\text { segundos }\end{array}$ & 3 & $11,54 \%$ & 22 & $84.62 \%$ & 1 & $3.85 \%$ & 10 & $20.41 \%$ & 39 & $79.59 \%$ & 0 & $0.00 \%$ & 0.26 \\
\hline $\begin{array}{l}\text { 10. No aterriza en el } \\
\text { mismo punto* }\end{array}$ & 0 & $0.00 \%$ & 20 & $76.92 \%$ & 6 & $23.08 \%$ & 0 & $0.00 \%$ & 48 & $97.96 \%$ & 1 & $2.04 \%$ & 0.00 \\
\hline
\end{tabular}

\section{DISCUSIÓN}

El objetivo del presente estudio fue cuantificar y describir los valores normales de la versión modificada del TJA en deportistas adolescentes de tecnificación. De forma secundaria se compararon los valores resultantes según sexo. Los principales hallazgos indicaron que el promedio puntuación global de la versión modificada del TJA de los participantes fue de $8.85 \pm 2.31$ sin apreciarse diferencias significativas entre $\operatorname{sexos}(p=0.42$; ES = 


\section{Detección de déficits neuromusculares en deportistas adolescentes}

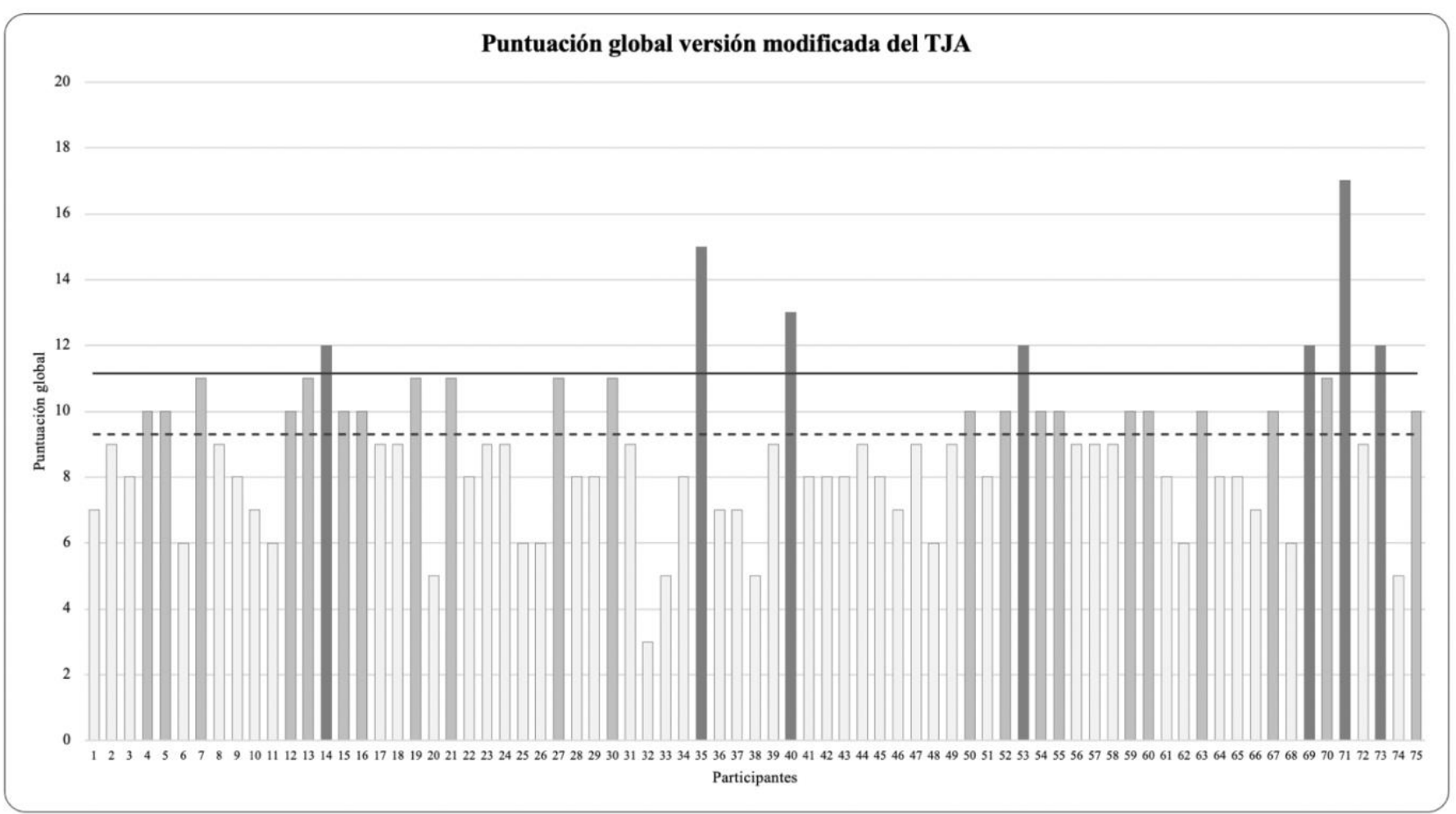

Figura 3. Puntuación global de la versión modificada del TJA. La línea discontinua marca el umbral de puntuaciones "pequeñas a moderadas" y la línea continua marca el umbral de puntuaciones "altas a extremas".

sexo de los participantes, se observaron diferencias significativas en los siguientes criterios: valgo de la extremidad inferior en el aterrizaje (criterio 1) $(\chi 2=$ $10.29 ; p<0.01)$, muslos no paralelos (criterio 2$)(\chi 2$ $=20.79 ; p<0.01)$, mala alineación de los pies con los hombros (criterio 4) $(\chi 2=22.59 ; p<0.01) \mathrm{y}$, no aterriza en el mismo punto (salto consistente) (criterio 10$)(\chi 2=8.88 ; p<0.01)$.

El TJA es un instrumento que permite identificar alteraciones neuromusculares, mediante el análisis del patrón técnico de salto y aterrizaje. La variable puntuación global, se obtiene a partir de la suma de las puntuaciones de los diez criterios y permite clasificar a los deportistas según la predisposición a sufrir una lesión del LCA. Recientemente, FortVanmeerhaeghe, et al. (2019), publicaron un estudio dónde analizaron el patrón de salto y aterrizaje en una muestra de deportistas adolescentes amateurs y sus resultados indicaron una puntuación global de $8.45 \pm$ 1.86 en hombres y $9.27 \pm 1.68$ en mujeres. Standing y Maulder (2019) también reportaron la puntuación global de la versión modificada del TJA en una muestra de deportistas adolescentes hombres (12.3 \pm
2.83). En el presente estudio, los resultados obtenidos de la puntuación global fueron de $8.85 \pm 2.63$ en hombres y, $8.86 \pm 2.14$ en mujeres, valores sensiblemente menores a los reportados por FortVanmeerhaeghe, et al. (2019), hecho que pudiera atribuirse a la naturaleza de la muestra del presente estudio, deportistas de un centro de tecnificación con un volumen de sesiones de entrenamiento superior a equipos amateurs de las mismas modalidades deportivas. Si bien la versión modificada del TJA (Fort-Vanmeerhaeghe, et al., 2017), no establece una puntuación específica umbral de riesgo de lesión del LCA, en la versión original, Myer et al., (2008a) sugirieron que una puntuación superior a seis (respecto a una valoración dicotómica de los diez criterios) debería ser objeto de un programa de prevención específico. En este sentido, en el presente estudio se incluyó el cálculo del Z-Score (Dos'Santos et al., 2020) con el fin de identificar a los participantes con puntuaciones más altas respecto al promedio del grupo y, clasificarlos en dos subgrupos para optimizar las estrategias de prevención de lesiones. 


\section{Benet-Vigo, A. Arboix-Alió, J., Montalvo, A.M., Myer, G.D. y Fort-Vanmeerhaeghe, A.}

Asimismo, resulta interesante destacar que, en los criterios 3) muslos no alineados durante la fase de vuelo, 5) posición de los pies no paralela y, 10) no aterriza en el mismo punto (salto consistente), ningún sujeto fue capaz de ejecutar la técnica de salto y aterrizaje sin alteraciones. Los dos primeros criterios se agrupan en la dominancia de la pierna como factor de riesgo neuromuscular y se asocian a asimetrías de la extremidad inferior en el patrón de salto y aterrizaje. Este hecho se puede atribuir a que los participantes del estudio practican deportes de naturaleza unilateral, donde la propia práctica contribuye a un desarrollo asimétrico de las extremidades, que imposibilita a los participantes no tener alteraciones en el patrón de salto y aterrizaje respecto a estos criterios (Maloney et al., 2017). En cuanto al criterio 10) no aterriza en el mismo punto (salto consistente), asociado a un déficit de fuerza en la musculatura estabilizadora del tronco (FortVanmeerhaeghe et al., 2019; Myer et al., 2011b), no fue posible mantener la consistencia de la recepción del salto en la marca durante la ejecución de la prueba, en ningún participante.

Profundizando en la comparativa de los resultados que obtuvieron significación según el sexo de los participantes, la presencia, ya fuese leve o severa, del criterio 1) valgo de la extremidad inferior, fue superior en las mujeres respecto a los hombres, llegando a porcentajes cercanos al $80 \%(n=38)$. Este déficit neuromuscular se atribuye a un déficit de fuerza de las extremidades inferiores que impide una correcta absorción de las fuerzas generadas en acciones deportivas y tiene mayor incidencia en mujeres (Ford et al., 2003; Hewett et al., 2005). Además, a menudo se acentúa por un mayor ángulo Q que predispone al valgo durante la fase de aterrizaje de un salto (McLean et al., 2005).

Una mala alineación de los pies con los hombros corresponde al criterio de puntuación 4 del TJA, también agrupado en la dominancia del ligamento como factor de riesgo neuromuscular de lesión, al igual que el valgo dinámico (Fort-Vanmeerhaeghe et al., 2017). Sin embargo, por lo que respecta a los resultados obtenidos en el presente estudio, los hombres presentaron más alteraciones del patrón de salto (leves: $77 \%$; severas: $15 \%$ ) respecto a las mujeres (inexistentes: 59\%; leves: 41\%). Este criterio va estrechamente relacionado con las medidas antropométricas de los sujetos, considerando la anchura de los hombros la referencia para medir la distancia entre los pies, por lo que resulta difícil justificar los resultados basándonos en déficits biomecánicos y, pudiendo atribuir dicha alteración, más presente en hombres, a una mayor anchura de hombros respecto a las mujeres.

Con relación a los dos otros criterios de puntuación que obtuvieron significación en la comparativa según sexo, ambos se asocian a la dominancia del tronco, como déficit neuromuscular (Fort-Vanmeerhaeghe et al., 2017). Tanto Zazulak et al., (2007) como, posteriormente, Myer et al., (2008b), apuntaron que un déficit de activación de la musculatura estabilizadora del tronco podía asociarse con un incremento de las cargas de abducción sobre la rodilla y con una mayor alteración propioceptiva, ambos factores más presentes en mujeres deportistas. En este sentido, el criterio de puntuación 2) muslos no paralelos (punto máximo de salto) reportó puntuaciones más altas en las mujeres respecto a los hombres. De hecho, fue uno de los criterios donde más mujeres presentaron una alteración severa del patrón de salto $(27 \% ; \mathrm{n}=13)$. Sin embargo, en el criterio de puntuación 10) no aterriza en el mismo punto (salto consistente), más hombres presentaron alteraciones severas del patrón de salto (23\%), respecto a las mujeres (2\%), aunque ninguno de los participantes fue capaz de ejecutar dicho criterio con corrección. Estos últimos resultados que apuntan a una menor estabilidad del tronco en hombres se podrían atribuir a una capacidad de salto superior alcanzando una mayor altura y por ende una mayor inestabilidad en el momento del aterrizaje. A pesar de que la altura del salto no sea una variable contemplada en el análisis cualitativo, podría influir en el resto de los criterios.

En cuanto a las limitaciones del presente estudio cabe señalar que el TJA y su versión modificada no son herramientas validadas pese a la buena fiabilidad demostrada en estudios anteriores (FortVanmeerhaeghe et al., 2017; Herrington et al., 2013; Read et al., 2016). Sin embargo, resulta una herramienta de gran utilidad ya que incluye, a través de su análisis cualitativo, la evaluación de déficits biomecánicos y neuromusculares asociados a los diez criterios del instrumento, mediante un contexto de fatiga que pretende asemejarse a la realidad competitiva, que otros instrumentos más analíticos no contemplan. También es importante mencionar que la 


\section{Detección de déficits neuromusculares en deportistas adolescentes}

muestra no fue equitativa en cuanto a sexo, dado que no tuvimos la posibilidad de reclutar más deportistas masculinos (jugadores de baloncesto).

Finalmente, y con el objetivo de evidenciar efectividad de los programas de entrenamiento neuromuscular con el fin de reducir la incidencia lesiva del LCA, se deberían llevar a cabo estudios longitudinales empleando la versión modificada del TJA como instrumento de evaluación pre y post intervención.

\section{CONCLUSIONES}

Los resultados obtenidos del análisis del patrón de salto y aterrizaje muestran que el promedio puntuación global de la versión modificada del TJA de los participantes fue de $8.85 \pm 2.31$, sin apreciar diferencias significativas entre sexos $(8.85 \pm 2.63$ en hombres vs. $8.86 \pm 2.14$ en mujeres). En la comparativa de los criterios según sexo, se obtuvieron diferencias significativas en cuatro criterios. En dos de ellos las mujeres obtuvieron puntuaciones más altas (1) valgo dinámico y 2) muslos no paralelos), mientras que en otros dos criterios fueron los hombres (4) mala alineación de los pies con los hombros y 10) no aterriza en el mismo punto).

\section{DECLARACIÓN DE CONFLICTO DE INTERESES}

Los autores declaran que no existe conflicto de intereses relevante para este estudio.

\section{REFERENCIAS}

1. Agel, J., Rockwood, T., y Klossner, D. (2016). Collegiate ACL Injury Rates Across 15 Sports: National Collegiate Athletic Association Injury Surveillance System Data Update (2004-2005 Through 2012-2013). Clinical Journal of Sport Medicine, 26(6), 518-523. doi:10.1097/JSM.0000000000000290

2. Arundale, A. J. H., Kvist, J., Hägglund, M., y Fältström, A. (2020a). Jump performance in male and female football players. Knee Surgery, Sports Traumatology, Arthroscopy, 28(2), 606613. doi:10.1007/s00167-019-05747-1

3. Arundale, A., Kvist, J., Hägglund, M., y Fältström, A. (2020b). Tuck jump score is not related to hopping performance or patientreported outcome measures in female soccer players. International Journal of Sports Physical Therapy, 15(3), 395-406.

4. Bahr, R., y Krosshaug, T. (2005). Understanding injury mechanisms: a key component of preventing injuries in sport. British Journal of Sports Medicine, 39(6), 324-329. doi:10.1136/bjsm.2005.018341

5. Crossley, K. M., Zhang, W. J., Schache, A. G., Bryant, A., y Cowan, S. M. (2011). Performance on the single-leg squat task indicates hip abductor muscle function. American Journal of Sports Medicine, 39(4), 866-873. doi:10.1177/0363546510395456

6. Dos'Santos, T., Thomas, C., y Jones, P. A. (2020). Assessing Interlimb Asymmetries. Strength y Conditioning Journal. doi.org/10.1519/ssc.0000000000000590

7. Fleiss, J.L. (1986). The Design and Analsys of Clinical Experiments. Applied Psychological Measurement. New York: Wiley and Son.

8. Ford, K R, Myer, G. D., y Hewett, T. E. (2003). Valgus knee motion during landing in high school female and male basketball players. Medicine and Science in Sports and Exercise, 35(10), $1745-1750$. doi:10.1249/01.MSS.0000089346.85744.D9

9. Fort-Vanmeerhaeghe, A., Benet, A., Mirada, S., Montalvo, A. M., y Myer, G. D. (2019). Sex and Maturation Differences in Performance of Functional Jumping and Landing Deficits in Youth Athletes. Journal of Sport Rehabilitation, 28(6), 606-613. doi:10.1123/jsr.2017-0292

10. Fort-Vanmeerhaeghe, A., Montalvo, A. M., Lloyd, R. S., Read, P., y Myer, G. D. (2017). Intra- and inter-rater reliability of the modified tuck jump assessment. Journal of Sports Science and Medicine, 16(1), 117-124.

11. Fort-Vanmeerhaeghe, A., Romero-Rodriguez, D., Montalvo, A. M., Kiefer, A. W., Lloyd, R. S., y Myer, G. D. (2016). Integrative Neuromuscular Training and Injury Prevention in Youth Athletes. Part I: Identifying Risk Factors. Strength and Conditioning Journal, 38(3), 36-48. doi:10.1519/SSC.0000000000000229

12. Fox, A. S. (2018). Change-of-Direction Biomechanics: Is What's Best for Anterior 


\section{Benet-Vigo, A. Arboix-Alió, J., Montalvo, A.M., Myer, G.D. y Fort-Vanmeerhaeghe, A.}

Cruciate Ligament Injury Prevention Also Best for Performance? Sports Medicine, 48(8), 17991807. Doרררi:10.1007/s40279-018-0931-3

13. Gornitzky, A. L., Lott, A., Yellin, J. L., Fabricant, P. D., Lawrence, J. T., y Ganley, T. J. (2016). Sport-Specific Yearly Risk and Incidence of Anterior Cruciate Ligament Tears in High School Athletes: A Systematic Review and Metaanalysis. American Journal of Sports Medicine, 44(10),

2716-2723. doi: $10.1177 / 0363546515617742$

14. Griffin, L. Y., Albohm, M. J., Arendt, E. A., Bahr, R., Beynnon, B. D., DeMaio, M., Dick, R. W., Engebretsen, L., Garrett, W. E., Hannafin, J. A., Hewett, T. E., Huston, L. J., Ireland, M. L., Johnson, R. J., Lephart, S., Mandelbaum, B. R., Mann, B. J., Marks, P. H., Marshall, S. W., y Yu, B. (2006). Understanding and preventing noncontact anterior cruciate ligament injuries: A review of the Hunt Valley II Meeting, January 2005. American Journal of Sports Medicine, 34(9),

1512-1532. doi: $10.1177 / 0363546506286866$

15. Herrington, L., Myer, G. D., y Munro, A. (2013). Intra and inter-tester reliability of the tuck jump assessment. Physical Therapy in Sport, 14(3), 152-155. doi: 10.1016/j.ptsp.2012.05.005

16. Hewett, T. E, Myer, G. D., Ford, K. R., Heidt, R. S., Colosimo, A. J., McLean, S. G., van den Bogert, A. J., Paterno, M. V., y Succop, P. (2005). Biomechanical measures of neuromuscular control and valgus loading of the knee predict anterior cruciate ligament injury risk in female athletes: a prospective study. The American Journal of Sports Medicine, 33(4), 492-501. doi:10.1177/0363546504269591

17. Hewett, T. E., Myer, G. D., y Ford, K. R. (2006). Anterior cruciate ligament injuries in female athletes: Part 1, mechanisms and risk factors. The American Journal of Sports Medicine, 34(2), 299-311. doi.org/10.1177/0363546505284183

18. Hewett, T. E., Torg, J. S., y Boden, B. P. (2009). Video analysis of trunk and knee motion during non-contact anterior cruciate ligament injury in female athletes: Lateral trunk and knee abduction motion are combined components of the injury mechanism. British Journal of Sports Medicine, 43(6), 417-422. doi:10.1136/bjsm.2009.059162
19. Hewett, T. E., Ford, K. R., Hoogenboom, B. J., y Myer, G. D. (2010). Understanding and preventing acl injuries: current biomechanical and epidemiologic considerations - update 2010. North American Journal of Sports Physical Therapy: NAJSPT, 5(4), 234-251.

20. Hewett, T. E., Ford, K. R., Xu, Y. Y., Khoury, J., y Myer, G. D. (2016). Utilization of ACL Injury Biomechanical and Neuromuscular Risk Profile Analysis to Determine the Effectiveness of Neuromuscular Training. American Journal of Sports Medicine, 44(12), 3146-3151. doi: $10.1177 / 0363546516656373$

21. Lindblom, H., Waldén, M., Carlfjord, S., y Hägglund, M. (2020). Limited positive effects on jump-landing technique in girls but not in boys after 8 weeks of injury prevention exercise training in youth football. Knee Surgery, Sports Traumatology, Arthroscopy, 28(2), 528-537. doi:10.1007/s00167-019-05721-x

22. Lohmander, L. S., Englund, P. M., Dahl, L. L., y Roos, E. M. (2007). The long-term consequence of anterior cruciate ligament and meniscus injuries: Osteoarthritis. American Journal of Sports Medicine (Vol. 35, Issue 10, pp. 17561769). SAGE PublicationsSage CA: Los Angeles, CA. doi:10.1177/0363546507307396

23. Maloney S.J., Richards J., Nixon D.G.D., Harvey L.J., y Fletcher I.M. (2017). Do stiffness and asymmetries predict change of direction performance? Journal of Sports Science. 35(6):547-556.

doi:10.1080/02640414.2016.1179775

24. McLean, S. G., Walker, K., Ford, K. R., Myer, G. D., Hewett, T. E., y Van Den Bogert, A. J. (2005). Evaluation of a two-dimensional analysis method as a screening and evaluation tool for anterior cruciate ligament injury. British Journal of Sports Medicine, 39(6), 355-362. doi:10.1136/bjsm.2005.018598

25. Mirwald, R. L., G. Baxter-Jones, A. D., Bailey, D. A., y Beunen, G. P. (2002). An assessment of maturity from anthropometric measurements. Medicine y Science in Sports y Exercise, 34(4), 689-694. doi: 10.1097/00005768-20020400000020 


\section{Detección de déficits neuromusculares en deportistas adolescentes}

26. Myer, G. D., Jayanthi, N., Difiori, J. P., Faigenbaum, A. D., Kiefer, A. W., Logerstedt, D., y Micheli, L. J. (2015). Sport Specialization, Part I: Does Early Sports Specialization Increase Negative Outcomes and Reduce the Opportunity for Success in Young Athletes? Sports Health, 7(5), 437-442. doi: 10.1177/1941738115598747

27. Myer, G. D., Faigenbaum, A., Chu, D. A., Falkel, J., Ford, K. R., Best, T., y Hewett, T. E. (2011a). Integrative Training for Children and Adolescents: Techniques and Practices for Reducing Sports-Related Injuries and Enhancing Athletic Performance. The Physician and Sportsmedicine, 39(1), 74-84. doi: 10.3810/psm.2011.02.1854

28. Myer, G. D., Ford, K. R., Khoury, J., Succop, P., y Hewett, T. E. (2011b). Biomechanics laboratory-based prediction algorithm to identify female athletes with high knee loads that increase risk of ACL injury. British Journal of Sports Medicine, 45(4), 245-252. doi:10.1136/bjsm.2009.069351

29. Myer, G. D., Ford, K. R., y Hewett, T. E. (2008a). Tuck jump assessment for reducing anterior cruciate ligament injury risk. Athletic Therapy Today, 13(5), 39-44. doi:10.1123/att.13.5.39

30. Myer, G. D., Chu D. A., Brent, J. L., y Hewett, T.E. (2008b). Trunk and hip control neuromuscular training for the prevention of knee joint injury. Clinics in Sports Medicine. 27:42548. doi:10.1016/j.csm.2008.02.006

31. Padua, D. A., Boling, M. C., Distefano, L. J., Onate, J. A., Beutler, A. I., y Marshall, S. W. (2011). Reliability of the landing error scoring system-real time, a clinical assessment tool of jump-landing biomechanics. Journal of Sport Rehabilitation, 20(2), 145-156. doi: 10.1123/jsr.20.2.145

32. Read, P. J., Oliver, J. L., De Ste Croix, M. B. A., Myer, G. D., y Lloyd, R. S. (2016). Reliability of the Tuck Jump Injury Risk Screening Assessment in Elite Male Youth Soccer Players. Journal of Strength and Conditioning Research, 30(6), 1510-1516. doi:10.1519/JSC.0000000000001260
33. Shimokochi, Y., y Shultz, S. J. (2008). Mechanisms of noncontact anterior cruciate ligament injury. Journal of Athletic Training, 43(4), 396-408. doi:10.4085/1062-6050-43.4.396

34. Smith, C. A., Chimera, N. J., Lininger, M. R., y Warren, M. (2017). Modified tuck jump assessment: Reliability and training of raters. Journal of Sports Science and Medicine (Vol. 16, Issue 3, pp. 440-441).

35. Standing, R., y Maulder, P. (2019). The Effectiveness of Progressive and Traditional Coaching Strategies to Improve Sprint and Jump Performance Across Varying Levels of Maturation within a General Youth Population. Sports, 7(8), 186. doi:10.3390/sports7080186

36. Sugimoto, D., Myer, G. D., Micheli, L. J., y Hewett, T. E. (2015). ABCs of Evidence-Based Anterior Cruciate Ligament Injury Prevention Strategies in Female Athletes. Physical Medicine and Rehabilitation Reports, 3(1), 43-49. doi:10.1007/s40141-014-0076-8

37. Yanguas-Leyes, J., Til-Pérez, L., y Cortés de Olano, C. (2011). Lesión del ligamento cruzado anterior en fútbol femenino. Estudio epidemiológico de tres temporadas. Apunts Medicina de l'Esport, 46(171), 137-143. doi:10.1016/j.apunts.2011.02.006

38. Zazulak B.T., Hewett T.E., Reeves N. P., Goldberg B, y Cholewicki J. (2007). Deficits in neuromuscular control of the trunk predict knee injury risk. A prospective biomechanicalepidemiologic study. American Journal of Sports Medicine. 35:1123-30. doi: $10.1177 / 0363546507301585$ 


\section{Benet-Vigo, A. Arboix-Alió, J., Montalvo, A.M., Myer, G.D. y Fort-Vanmeerhaeghe, A.}

\section{Anexo 1: Propuesta práctica: ampliación del procedimiento}

Descripción y puntuación de los ítems valorados por el TJA modificado

\section{Acción de las rodillas y los muslos}

Ítem 1. Valgo de la extremidad inferior en el aterrizaje. Puntuamos " 0 " cuando en la fase de contacto con el suelo, las rodillas mantienen una correcta alineación fisiológica con los tobillos y la cadera y no se aprecia valgo dinámico. Cuando se produce dicho valgo dinámico puntuamos con un "1" siempre y cuando no haya contacto entre las rodillas. Cuando en la recepción del salto las rodillas contactan entre sí puntuamos con un "2".

Ítem 2. Muslos no paralelos (punto máximo de salto). Puntuamos " 0 " cuando, durante la fase de vuelo, en el punto máximo de salto, las rodillas se muestran al mismo nivel o por encima del muslo. Se considera alteración del patrón técnico de salto cuando las rodillas no asumen dicha altura. Se puntúa "1" si las rodillas están sensiblemente por debajo del punto medio del muslo (trazando una línea desde el punto medio del trocánter hasta el punto medio de la rótula). Cuando las rodillas están claramente por debajo de los glúteos se puntúa con un " 2 ".

Ítem 3. Muslos no alineados en la fase de vuelo. Puntuamos "0" cuando la línea trazada desde el punto medio de la rótula de ambas rodillas es paralela al suelo. Cuando una rodilla está por encima de la otra en tres o más ocasiones se considera alteración del patrón técnico de salto. Si esta desalineación no supera la línea de la tuberosidad tibial anterior se puntúa " 1 " y en caso contrario se indica un " 2 ".

\section{Acción de los pies durante la fase de contacto}

Ítem 4. Mala alineación de los pies con los hombros. Para iniciar la prueba se indica al sujeto que se sitúe dentro de la marca como indica la figura 1. Durante la ejecución los pies deben estar alineados con los hombros. Puntuamos " 0 " si se respeta dicha alineación manteniendo la misma distancia o superior entre el punto medio de la articulación glenohumeral y el punto medio del hueso astrágalo (tobillo). Si la distancia entre el punto medio del astrágalo de ambas extremidades es inferior al del punto medio de articulación glenohumeral se puntúa " 1 ", siempre que no haya contacto entre los pies. En el caso de que los pies contacten se considera una alteración grave del patrón de salto y se puntúa con un " 2 ".

Ítem 5. Posición de los pies no paralela (de la punta al talón). Puntuamos "0" cuando la posición de los pies en el momento de contacto es paralela durante todos los saltos que se realizan a lo largo de la prueba, tomando como referencia las marcas del rectángulo de salto. Consideramos una alteración leve del patrón técnico de salto, puntuando " 1 ", cuando los pies no contactan en paralelo, pero la distancia entre los talones no supera la mitad del pie, dejando al descubierto el maléolo del pie distal del plano sagital. Cuando la distancia entre talones supera la mitad del pie se considera una alteración severa del patrón técnico de salto y se puntúa con un " 2 ".

Ítem 6. Contacto desigual de los pies (aterrizaje asimétrico). Puntuamos " 0 " cuando el contacto con el suelo es con los dos pies a la vez, analizando el vídeo fotograma a fotograma. Se considera una alteración moderada del patrón de salto, puntuando con un "1", pero no supera los 250 milisegundos. Cuando el tiempo de contacto entre los dos pies es superior a los 500 milisegundos puntuamos " 2 ".

Ítem 7. Demasiado ruido en el aterrizaje. Puntuamos "0" cuando en la fase de contacto con el suelo, el sujeto solo contacta con las puntas de los pies (metatarsos) y apenas se aprecia ruido en el contacto. Si el sujeto contacta con los dos tercios de la superficie del pie, sin llegar a contactar con los talones y el ruido es audible, puntuamos " 1 ". Puntuamos "2" si existe ruido fuerte y pronunciado al aterrizar y el sujeto aterriza con toda la superficie del pie.

\section{Análisis de la técnica pliométrica del salto}

Ítem 8. Pausa entre saltos. Puntuamos "0" cuando el tiempo de contacto con el suelo es inferior a los 250 milisegundos. Puntuamos "1" si el tiempo de contacto es entre 250 y 500 milisegundos. Puntuamos " 2 " si el tiempo de contacto es superior a los 500 milisegundos.

Ítem 9. La técnica empeora antes de los 10 segundos. Puntuamos "0" cuando el sujeto es capaz de ejecutar la prueba completa sin alterar el patrón individual de 
salto. Cuando la fatiga aparece a partir del sexto segundo, alterando alguno de los criterios, puntuamos "1" y si dicha alteración se produce antes, puntuamos "2".

Ítem 10. No aterriza en el mismo punto (salto consistente). Para iniciar la prueba se indica a al sujeto que se sitúe dentro de la marca como indica la figura 1. Para puntuar " 0 " el sujeto debe aterrizartodos los saltos con los pies en la posición inicial de la prueba. Puntuamos "1" cuando se altera el punto de recepción del salto, pero el sujeto aterriza dentro del rectángulo de salto. Se considera una alteración grave del patrón de salto cuando el sujeto aterriza completamente fuera del rectángulo de salto se puntúa " 2 ".

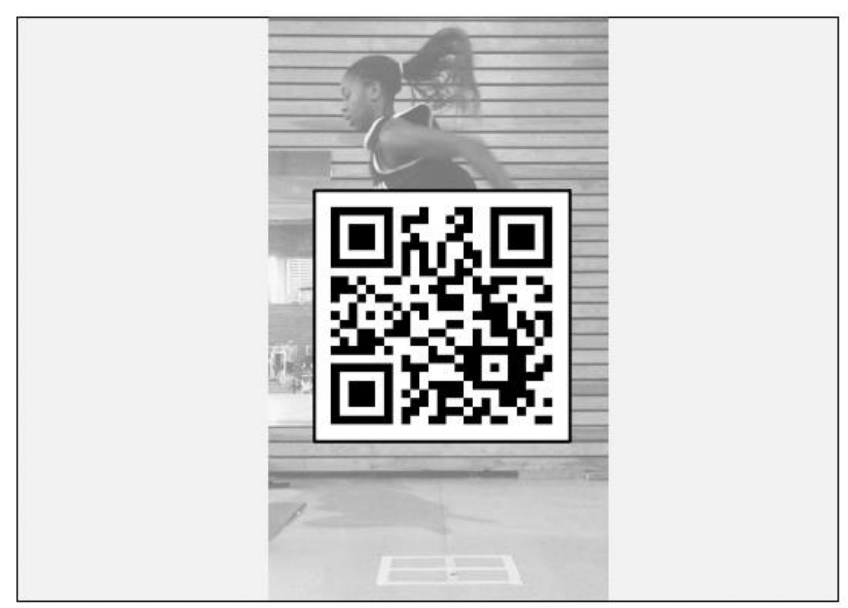

Figura 4. Descripción videográfica de los criterios de puntuación de la propuesta modificada del TJA. 
Benet-Vigo, A. Arboix-Alió, J., Montalvo, A.M., Myer, G.D. y Fort-Vanmeerhaeghe, A.

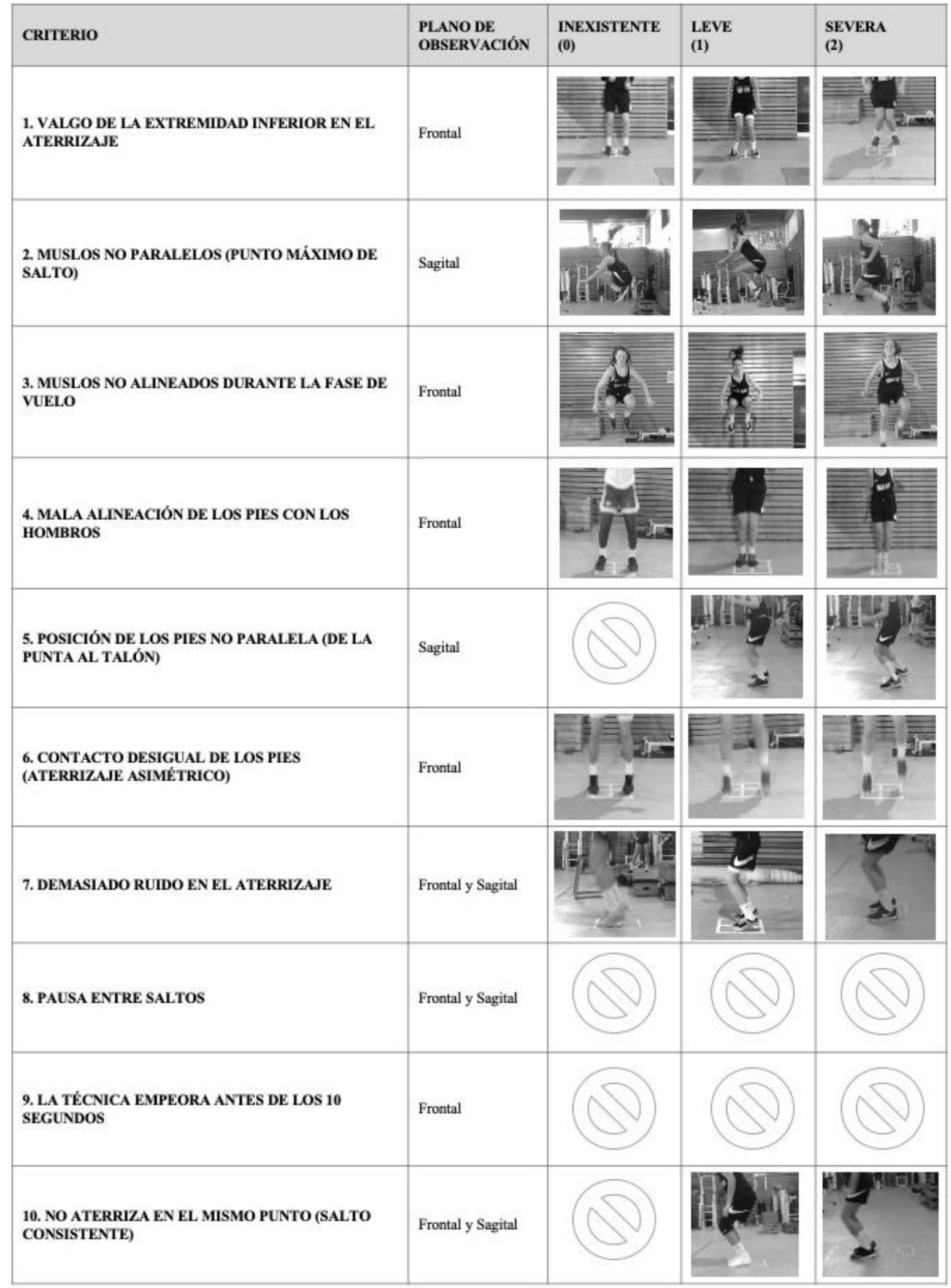

Figura 5. Criterios de puntuación de la propuesta modificada del Tuck Jump Assessment (adaptado de Fort-Vanmeerhaeghe y col., 2019). 